\title{
Tratamiento del paciente pediátrico con COVID-19
}

\section{COVID-19 treatment for pediatric patients}

\author{
Mónica E. Rosales-Salán ${ }^{1 *}$, Aída G. Barrera-Pérez², Magda F. Velásquez-Tohom³ \\ ${ }^{1}$ Unidad Didáctica de Farmacología, ${ }^{2}$ Centro de Investigaciones de las Ciencias de la Salud (CICS), \\ ${ }^{3}$ Dirección de Investigación, Facultad de Ciencias Médicas, Universidad de San Carlos de Guatemala
}

*Autor al que se dirige la correspondencia: mrosales2657@medicina.usac.edu.gt

Recibido: 29 de julio 2020 / Revisión: 14 de septiembre 2020 / Aceptado: 20 de octubre 2020

Resumen

$\mathrm{C}$ OVID-19 afecta entre el 1.2 al 5\% de niños y adolescentes en diversos países incluyendo Guatemala, la mayoría con manifestaciones clínicas leves a moderadas. La terapéutica farmacológica para enfermedad grave aún no está completamente establecida, por lo que se realizó una revisión de literatura de artículos científicos publicados en revistas indizadas para proporcionar una actualización del tratamiento de COVID-19 en niños y adolescentes, que ayude a orientar las decisiones clínicas. El tratamiento de casos leves consiste en antipiréticos, antibióticos en neumonía leve; en casos moderados, monitoreo de signos vitales, cánula binasal, manejo de líquidos, antipiréticos y analgésicos, toma de hemocultivo, inicio de antibióticos y uso de beta agonistas de acción corta o esteroides inhalados. En enfermedad grave, ventilación mecánica no invasiva o invasiva, ventilación prono temprana, soporte nutricional, hídrico y vasopresor, mantenimiento del equilibrio ácido base y de electrolitos. Se indica el uso de antibióticos y antivirales como remdesivir y el manejo inmunológico con interferón alfa, esteroides y/o inmunoglobulina. Se monitorea función hepática, renal, inmunológica, hematológica y cardíaca. No se ha encontrado evidencia de que la hidroxicloroquina, ivermectina y algunos antivirales disminuyan la mortalidad en pacientes pediátricos. Actualmente no existe ningún medicamento específico, validado científicamente para el tratamiento de COVID-19 en niños. El tratamiento para casos graves se decide a partir de las experiencias del tratamiento en adultos, por lo cual es importante continuar con investigación de fármacos específicos para la población pediátrica.

Palabras claves: Infecciones por coronavirus, terapéutica, tratamiento farmacológico, enfermedades transmisibles, niño, adolescente, pediatría

Abstract

COVID-19 affects among 1.2 to $5 \%$ of children and adolescents in various countries, counting Guatemala, the most with mild to moderate clinical manifestations. Pharmacological therapy for severe disease has not yet been fully established. A literature review of scientific articles from indexed journals was conducted to provide an update on the treatment of COVID-19 in children and adolescents, to help guide clinical decisions. Treatment of mild cases consists of antipyretics and antibiotics for pneumonia. In moderate cases is recommended to monitor vital signs, to obtain blood cultures and to treat with binasal cannula, fluids, antipyretics and analgesics, short-acting beta agonists and/or inhaled steroids and antibiotics when needed. In severe illness, treatment includes non-invasive or invasive mechanical ventilation, early prone ventilation, nutritional, hydration and vasopressor support, maintenance of acid-base, and electrolyte balance. The use of antibiotics and antivirals as remdesivir is recommended, as well as the use of immunomodulators such as interferon alfa, steroids and/or immunoglobulin. To monitor patient's evolution; liver, kidney, immunological, hematological, and cardiac function must be assessed. No evidence has been found that hydroxychloroquine, ivermectin and other antivirals decrease mortality in pediatric patients. Currently, there is no specific, scientifically validated medication for the treatment of COVID-19 in children. Treatment for severe cases is decided based on management experiences in adults, so it is important to doing research on specific drugs for the pediatric population.

Keywords: Coronavirus Infections, Therapeutics, Drug Therapy, Transmissible diseases, Child, Adolescent, Pediatrics 


\section{Introducción}

La pandemia relacionada a COVID-19 o SARSCoV-2 ha tenido impacto social, económico y sobre todo en el ámbito de salud a nivel mundial, tanto por la tasa de mortalidad como por el desborde de la capacidad de atención en adultos, ya que se estima que los casos se duplican en número cada cuatro días (Uddin et al., 2020), causando el cierre incluso de ciudades y naciones como medida en pro de la contención del contagio (She et al., 2020).

A pesar del alto número de casos en adultos, la incidencia es de alrededor de $1-5 \%$ en menores de 19 años (Ludvigsson 2020; Rehman et al., 2020) a nivel mundial y $1 \%$ a nivel nacional (Ministerio de Salud Pública y Asistencia Social [MSPAS], 2020).

El agente causal es un virus ARN con genoma no fragmentado de 29,903 kb; relacionado con los causantes del síndrome respiratorio agudo grave (SARS por sus siglas en inglés) y del síndrome respiratorio de oriente medio (MERS por sus siglas en inglés) (Lin et al., 2020) que interactúa con las células que poseen receptores de la enzima convertidora de angiotensina 2 (ACE2 por sus siglas en inglés) (Uddin et al., 2020) y en el caso específico de las células de la inmunidad con aquellas que poseen la molécula de adhesión intercelular específica de las células dendríticas 3 y su proteína relacionada (Yuki et al., 2020).

El diagnóstico se realiza con la prueba de reacción en cadena de la polimerasa en tiempo real (PCR-RT por sus siglas en inglés) de hisopado nasofaríngeo (Mantovani et al., 2020; de Souza et al., 2020). En general los niños son asintomáticos o con manifestaciones clínicas leves, aunque alrededor del $8 \%$ de los pacientes pueden llegar a requerir ingreso a terapia intensiva (Götzinger, et al., 2020) debido al síndrome inflamatorio multisistémico, síndrome de dificultad respiratoria aguda o shock (Castagnoli et al., 2020; Li et al., 2020; Liguoro et al., 2020; Lin et al., 2020; Yong et al., 2020).

Al momento, si bien los casos leves únicamente requieren tratamiento con analgésico, antipirético e hidratación, los moderados presentan un reto en el uso apropiado de las medidas de sostén y monitoreo para evitar la progresión, mientras se disminuye el potencial de diseminación del germen al ambiente y personal de salud (uso de sistema de aporte de oxígeno en bajo y alto flujo, nebulizaciones) así como el uso racional de antibiótico para el tratamiento de coinfecciones adquiridas en la comunidad, que representa también un reto significativo (Calvo et al., 2020; Sankar et al., 2020).
En el caso de los pacientes con manifestaciones graves, el conflicto es mayor, ya que no existe al momento un tratamiento específico (Liguoro et al., 2020). Por lo que en general se extrapola de los estudios en adultos, entrando en conflicto con los datos de farmacocinética y farmacodinamia, propios de la edad pediátrica.

En dicha línea de pensamiento, se realiza una revisión de literatura de publicaciones científicas en revistas indizadas e informes técnicos que permita a los equipos multidisciplinarios tomar decisiones científicamente avaladas, estimulando la estandarización de clasificación y terapéutica, permitiendo el adecuado seguimiento de casos y favorecer el buen pronóstico de los mismos (Riggioni et al., 2020).

\section{Contenido}

\section{Epidemiología}

Los coronavirus son patógenos que atacan al ser humano, provocando enfermedades principalmente del tracto digestivo y respiratorio, afectando a personas de todas las edades (Rothan \& Byrareddy 2020). Las manifestaciones clínicas causadas por la infección del nuevo coronavirus SARS-CoV-2 en pacientes pediátricos, han sido poco descritas y diferenciadas para los diferentes grupos de edad (Hong et al., 2020).

Han pasado más de 7 meses desde el inicio de la pandemia provocada por el SARS-CoV-2 y todavía existe poca información sobre la incidencia de la infección en niños. Datos de Europa obtenidos a través de un estudio multicéntrico que incluyó información de 454 menores de 18 años en 21 países y 77 centros clínicos, mostró que en general los niños tienen manifestaciones leves, aunque el $8 \%$ presentó manifestaciones severas con ingreso a unidades de cuidados intensivos y ventilación prolongada. Además encontró una razón de 1.15 niños por cada niña (Götzinger et al., 2020). La frecuencia de casos de COVID-19 en niños que se ha reportado es $2 \%$ en China, $1.2 \%$ en Italia y $5 \%$ en Estados Unidos (Ludvigsson, 2020).

En Guatemala los datos del Ministerio de Salud Pública y Asistencia Social muestran que los niños de $0-9$ años representan alrededor del $1 \%$ de todos los casos y los de 10-20 años el 4\%. El sexo masculino representa el $52 \%$ de los niños de $0-9$ años y el 55\% del grupo de 10-20 años. La mortalidad del grupo total de 0-20 años, es alrededor de 1\% (MSPAS, 2020). De acuerdo con el Centro de Control y Prevención de la 
Enfermedad de Europa, la tasa de seropositividad en niños de 11-20 años se encontró en valores de 1-1.2\% en Italia y $0 \%$ en Islandia de 848 niños menores de 10 años examinados. Sin embargo, en población sintomática, el $6.7 \%$ positivos eran menores de 10 años (European Centre for Disease Prevention and Control [ECDC], 2020a).

La transmisión de persona a persona es el mecanismo de infección a través de las gotas respiratorias $>5 \mu$. En China se identificó que la infección en los niños fue secundaria al contacto cercano con adultos con síndrome respiratorio agudo COVID-19 (89\%) o a la exposición a áreas comunitarias epidémicas (33\%) (Qiu et al., 2020; Zimmermann \& Curtis, 2020). La transmisión placentaria se sospechó en un neonato que a las $30 \mathrm{~h}$ de vida fue positivo para COVID-19. No se ha demostrado el contagio a través de la leche materna (Rasmussen et al., 2020). Muestras de placenta de embarazadas con COVID-19 han resultado positivas por RT-PCR con resultados negativos en los neonatos (ECDC, 2020b).

Se considera que los niños pueden jugar un papel importante en la transmisión comunitaria del virus SARS-CoV-2, debido a que los cuadros de infección respiratoria aguda del tracto superior son prolongados y también a la excreción del virus en heces fecales. La transmisión respiratoria, oral y fecal podrían ser una preocupación en guarderías, escuelas y el hogar (Cruz \& Zeichner, 2020), aunque todavía es incierto si la detección del virus por RT-PCR en las heces fecales representa una replicación viral activa o material viral genómico residual (Balasubramanian et al., 2020).

Se ha descrito que el periodo de incubación de SARS-CoV-2 es de 1-14 días, más comúnmente de 3-7 días. En los niños se han encontrado periodos similares (She et al., 2020) y se recuperan en 1-2 semanas (Shen et al., 2020).

\section{Etiología y fisiopatología}

SARS-CoV-2 es un coronavirus similar a los causantes de SARS y MERS. Es un virus ARN con envoltura, de cadena simple en sentido positivo, con genoma no fragmentado de $29,903 \mathrm{~kb}$; el cual codifica 16 proteínas no estructurales necesarias para su replicación y cuatro proteínas estructurales incluida la de la envoltura (E), la de membrana (M), de nucleocápside (N) y glicoproteína espiculada (S), esta última permite la subtipificación. Comparte el $75-80 \%$ del genoma con SARS-CoV hallado en murciélagos, que son los reservorios primarios, mientras que los hospederos intermediarios son los pangolines. Se han detectado mutaciones en el gen que codifica la proteína $\mathrm{S}$ y en el dominio de unión al receptor (receptor-binding domain $R B D$ ), que determina la posibilidad de invasión al hospedero y el tropismo celular (Lega et al., 2020; Li et al., 2020; Uddin et al., 2020).

La proteína $S$ posee dos subunidades funcionales: S1 encargada de la unión a la célula del hospedero y S2 a cargo de la fusión de membranas viral y celular. Esto a través de su receptor funcional ACE2 presente en pulmón, corazón, íleo y riñón (Dhochak et al., 2020; Yuki et al., 2020).

Las células de la inmunidad innata como macrófagos y células dendríticas tienen expresión limitada para ACE2; para poder ser infectadas requieren de la unión del virus a la molécula de adhesión intercelular específica de las células dendríticas 3 (dendritic-cell specific intercellular adhesion molecule-3-grabbing nonintegrin [DC-SIGN]), y su proteína relacionada (DC-SIGN-related protein (DC-SIGNR, L-SIGN) (Yuki et al., 2020)

El ARN viral es reconocido por los receptores tipo Toll (TLR por sus siglas en inglés) TLR7/8, TLR3, llevando a la producción de interferón-1 (IFN-1 por sus siglas en inglés) a través de MyD88 e IRF7, expresión de moléculas de coestimulación de célula $\mathrm{T}$ (mediante TRAF6 e IRF3) y la producción de moléculas proinflamatorias (vía p38MAPK y AP-1). COVID-19 produce una preponderancia de estas últimas, según los hallazgos de incremento de citocinas como interleucinas (IL1b, IL-6) y quimiocinas (CCL2, CCL3, CCL5, CXCL8, CXCL10), compatibles con el síndrome de activación del macrófago y las lesiones observadas a nivel epitelial y endotelial que conducen a la falla orgánica múltiple (Li et al., 2020; Lin et al., 2020; Tian \& Ye, 2020). En el caso de los niños, también debe analizarse la función de las células plasmáticas generadas por las células B residentes en el timo a nivel del espacio perivascular CD138(+), las cuales secretan primordialmente IgG3 e IgG1 asociadas con la inmunidad a infecciones virales comunes y que pueden proveer inmunidad cruzada en este grupo etario (Dhochak et al., 2020; Felsenstein et al., 2020; Rehman et al., 2020; Yuki et al., 2020).

La tormenta de citoquinas como IL-6, IL-1 $\beta$, IL12, IL-18, IL-33, interferón- $\alpha$ (IFN- $\alpha$ ), IFN- $\gamma$ y factor de necrosis tumoral $\alpha$ (TNF- $\alpha$ ), quimiocinas como el ligando de quimiocina 2 (CCL-2), CCL-3, CCL-5 y los receptores de quimiocinas C-X-C 8. 9 y 10 (CXCL-8, CXCL-9, CXCL-10), se asocian con la activación del macrófago hacia el fenotipo (M1) y linfocitos T (Th1). 
Esta activación con retroalimentación positiva en las células de la inmunidad innata se asocia a la liberación de TNF- $\alpha$, proteína inflamatoria de macrófagos 1A (MIP-1A), proteína quimioatrayente de monocitos-1 e IL-7, factor estimulante de colonia de granulocitos (G-CSF, por sus siglas en inglés) y proteína quimiotrayente de monocitos 1 (MCP1, por sus siglas en inglés), las que se encuentran elevadas en relación a la gravedad del cuadro clínico presente. Otro aspecto que considerar es la observación de células CD4+ con expresión de interferón gama (IFN- $\gamma$ ) y la producción de IL8 por las células epiteliales invadidas que actúa como un quimioatrayente, tanto de neutrófilos como de células T. Así también, la mayor expresión de CD69, CD38 y CD44 habla de la activación excesiva de linfocitos T CD4+ y CD8+ lo que conduce a su agotamiento, evidente por el mayor porcentaje de los receptores Tm3+PD-1+ y NK grupo 2 miembro A (NKG2A) (Castagnoli et al., 2020; Dixon et al., 2020; Li et al., 2020; Lin et al., 2020; Moore \& June, 2020; Wang \& Ma, 2008; Yuki et al., 2020).

En relación a la fisiopatología de lesión orgánica, la lesión miocárdica inducida por SARS-CoV-2 se plantean tres hipótesis; (1) la lesión directa al miocardio, como en el caso de otros coronavirus (identificación de ARN viral en los miocitos hasta en el 35\% de sujetos infectados), (2) lesión asociada a la hipoxia tisular (desequilibrio entre disponibilidad y demanda de oxígeno miocárdico), la generación de radicales libres y la peroxidación de los lípidos de membrana y (3) la respuesta inflamatoria y la depresión de miocardio relacionada a los mediadores como IL-1 $\beta$, IFN- $\gamma$, IP-10, MCP-1 y linfocitos Th1 (Sanna et al., 2020).

Otros aspectos bajo investigación en relación a la patogénesis son; (1) la expresión de ACE2 en células linfoides innatas (ILC) tipos 2 y 3, (2) el papel de la activación de las células endoteliales, las cuales también expresan ACE2, (3) en el perfil protrombótico la pérdida de la integridad microvascular y mayor invasión viral (Castagnoli et al., 2020; Henry et al., 2020; Jin et al., 2020; Yuki et al., 2020) y (4) la regulación de ACE2 en la expresión del transportador de aminoácidos B0AT1, que disminuye la absorción de triptófano a nivel intestinal, necesario para la expresión de ARN mensajero de péptidos antimicrobianos (He et al., 2020).

En relación a la evasión inmune de SARS-CoV-2, se plantean hipótesis como la inhibición temprana del IFN-1 en las células infectadas de forma directa e indirecta. Además, altera la función normal de las células de la inmunidad innata (macrófagos, células dendrí- ticas, células NK) y adaptativa (linfocitos T CD4 y CD8) polarizándolas hacia las formas menos eficientes en infección (Th2, M2). Por otra parte, se considera la alteración en la activación del sistema inmune adaptivo por la presentación de antígeno vía MHC clase I/II y la mutación viral que puede guiar a una estimulación crónica y agotamiento de la respuesta de la célula $\mathrm{T}$ así como estados de hiperinflamación relacionada a la actividad persistente de los monocitos/mononucleares (Felsenstein et al., 2020; Gasparyan et al., 2020; Lega et al., 2020; Maggi et al., 2020).

\section{Diagnóstico}

En neonatos hijos de madres con diagnóstico de infección por SARS-CoV-2 (con o sin síntomas) se recomienda realizar la primera prueba $24 \mathrm{~h}$ después del nacimiento. Si la prueba es negativa, se debe repetir a las 48 h. Si existe sospecha de infección materna, la prueba al neonato se realiza solamente si presenta síntomas. Si es asintomático, se hará la prueba si el resultado de la madre resulta positivo. La prueba recomendada es PCR-RT, con una muestra de nasofaringe, orofaringe o recto. No se recomienda el uso de pruebas serológicas (Centers for Disease Control and Prevention [CDC], 2020a; Naranje et al., 2020; Velásquez-Gómez et al., 2020).

En niños y adolescentes el diagnóstico se realiza al igual que en los adultos, a través de hisopados nasofaríngeos u orofaríngeos con la prueba de PCR-RT (de Souza et al., 2020).

\section{Manifestaciones clínicas}

Los síntomas más frecuentes en los neonatos infectados con SARS-CoV-2 son fiebre, taquipnea, tos y vómitos. Las manifestaciones severas o críticas de la enfermedad se han descrito más en recién nacidos pretérmino y de bajo peso al nacer o en hijos de madres con diagnóstico de COVID-19 (Durán et al., 2020; Gil et al., 2020; Velásquez-Gómez et al., 2020). Los hallazgos radiológicos incluyen imágenes de neumonía, aumento de la trama pulmonar, engrosamiento o sombras nodulares de alta densidad. Los resultados de laboratorio muestran alteraciones menores e inespecíficas, pero en enfermedad severa, se alteran las enzimas hepáticas (Durán et al., 2020).

En una revisión sistemática que incluyó 1,117 niños, se detectó que $14.2 \%$ fueron asintomáticos (de Souza, 2020). De los niños enfermos $36.3 \%$ se cla- 
sificaron con enfermedad leve, $46 \%$ moderada, $2.1 \%$ severa y $1.2 \%$ crítica. Las manifestaciones leves son más frecuentes en menores de 5 años, las moderadas y severas en menores de 1 año y en mayores de 9 años. El riesgo de enfermedad severa o crítica es mayor en niños con inmunodepresión, cardiopatías, patología respiratoria crónica (como asma o fibrosis quística) y otras como diabetes mellitus tipo I, insuficiencia renal, síndrome del intestino corto y tratamiento con diálisis (de Souza et al, 2020.,Gil et al., 2020; Qiu et al., 2020; World Health Organization [WHO], 2020a).

En la revisión de los autores, de Souza y colaboradores (2020) se encontró que, a diferencia de los adultos, los niños presentan menos anormalidades de laboratorio y la linfopenia no es un signo patognomónico de la enfermedad. El 63\% de tomografías de pulmón reveló anormalidades, principalmente opacidades en vidrio esmerilado, sombras irregulares y consolidaciones. Solo se informó de una muerte (de Souza et al., 2020). En la Tabla 1 se presentan las manifestaciones clínicas, según la gravedad de la enfermedad.

Los resultados de una revisión sistemática conducida por Mantovani y colaboradores (2020) y un metaanálisis de Götszinger y colaboradores (2020), condujeron a las mismas conclusiones: en los adolescentes, al igual que en los niños, la COVID-19 se manifiesta de forma leve y tiene un buen pronóstico; el $47 \%$ de 2,855 pacientes incluidos, presentó fiebre, $37 \%$ tos, $4 \%$ diarrea, $2 \%$ congestión nasal y $1 \%$ disnea. El $79 \%$ presentó síntomas leves y $4 \%$, enfermedad crítica.

El trastorno inflamatorio multisistémico resulta de la liberación excesiva de citoquinas proinflamatorias y se relaciona con los datos de lesión endotelial, pulmonar y cardiovascular observado en el paciente críticamente enfermo (Lotfi et al., 2020).

Otro hallazgo es la aparición de lesiones cutáneas secundarias a la infección por SARS CoV-2. Gottlieb y Long (2020) identificaron los tipos de lesiones cutáneas más frecuentes en niños y adolescentes: exantema maculopapular, urticaria (19\%), vesículas, petequias, púrpura, eritema multiforme, tumefacciones $(19 \%)$ y menos frecuente, livedo racemosa e isquemia distal que puede acompañarse de necrosis. En la mayoría de los casos, las lesiones se manifiestan después de los síntomas respiratorios, pero si los preceden, podría indicar riesgo de complicaciones (Wollina et al., 2020).

\section{Tratamiento}

Se hace la anotación que aún no hay un medicamento específico y científicamente validado, aproba- do por la Organización Mundial de la Salud (OMS)/ Administración de Alimentos y Medicamentos de los Estados Unidos (FDA por sus siglas en inglés) para el tratamiento en niños (Liguoro et al., 2020). Varios estudios sobre antivirales, hidroxicloroquina y otros no han demostrado mejoría en la ya baja tasa de mortalidad en pediatría a nivel mundial $(0.0018 \%)$ (Patel, 2020). El uso de plasma de paciente convaleciente no tiene un efecto como factor independiente en la recuperación (Nguyen et al., 2020) e ivermectina no cuenta con publicaciones en revistas indizadas.

Se realiza una descripción de las opciones terapéuticas propuestas en pacientes pediátricos según su nivel de severidad (Tablas 2 y 3 ).

Algunos investigadores están proponiendo la inclusión de población pediátrica en ensayos clínicos de terapias para el tratamiento de COVID-19, para que las dosis que se empleen para tratarlos no sean producto de una extrapolación de las dosis de los adultos, sino que tomen en cuenta el metabolismo y la fisiopatología de los menores (Hwang et al., 2020). Algunas de las terapéuticas en investigación son las enlistadas en la Tabla 4.

Al momento de esta revisión, se toman como criterios de egreso para pacientes hospitalizados la resolución de los síntomas y signos clínicos, así como de la lesión radiológica pulmonar y el aclaramiento virológico en dos muestras con $24 \mathrm{~h}$ de diferencia en la toma (Sankar et al., 2020).

\section{Pronóstico}

En general, los niños se han presentado asintomáticos o con manifestaciones clínicas leves teniendo recuperación favorable en alrededor de 2 semanas (Riggioni et al., 2020). En aquellos que requirieron atención en cuidados intensivos, se ha evidenciado la necesidad de estandarizar criterios para recolectar información epidemiológica y clínica que permita un adecuado seguimiento de casos (WHO, 2020b).

\section{Vacunas}

La pandemia COVID-19 representa el problema de salud pública más serio asociado a una infección viral respiratoria desde 1918, sin que se cuente todavía con una vacuna específica y eficaz contra SARS-CoV-2 (Safadi-Palazzi, 2020). Actualmente la WHO (2020b), registra 23 ensayos clínicos de vacunas que se encuentran en diferentes fases y otros más en fase preclínica (WHO, 2020c). 
Tabla 1

Manifestaciones clínicas de COVID-19 en niños

\section{Leves}

Síntomas respiratorios superiores: congestión faríngea, dolor de garganta y fiebre, de corta duración.

Puede haber mialgia, tos, secreción nasal y estornudos.

En algunos casos no hay fiebre y solo se presentan síntomas digestivos como náusea, vómitos, dolor abdominal y diarrea.

Al examen físico se detecta congestión faríngea, sin anormalidades a la auscultación.

Sin anormalidades radiológicas.

Hallazgos de laboratorio: poco frecuentes linfopenia y elevación de la proteína $\mathrm{C}$ reactiva.

\section{Moderadas}

Neumonía leve.

Fiebre, tos seca al inicio, que luego se vuelve productiva, algunos pueden tener sibilancias.

Puede haber fatiga, dolor de cabeza y mialgia.

Al examen físico no se detecta fatiga ni hipoxemia, pero podrían auscultarse roncus o estertores secos y/o húmedos.

Lesiones pulmonares subclínicas en TAC de algunos pacientes asintomáticos y sin signos clínicos.

Hallazgos de laboratorio: linfopenia, elevación de la proteína $\mathrm{C}$ reactiva y de la procalcitonina.

\section{Severas}

A partir de síntomas tempranos como fiebre y tos que pueden acompañarse de síntomas gastrointestinales, la enfermedad progresa en alrededor de una semana hacia disnea y cianosis central:

- $\quad$ Taquipnea (> 70 respiraciones/min en $<$ de 1 año; $\geq 50$ respiraciones/ $\min$ en $>1$ año; $\mathrm{SO}_{2}<92 \%$ ), hipoxia y cianosis.

- Pérdida de consciencia, letargia, convulsiones, coma.

- Deshidratación, dificultad para alimentarse, disfunción gastrointestinal.

- Daño al miocardio.

- Lesiones a órganos blanco manifestadas por coagulopatías, rabdomiólisis y otras.

- Hallazgos de laboratorio: los enumerados en las manifestaciones leves y moderadas + elevación de creatincinasa $\mathrm{MB}$, procalcitonina y dímero D (relacionadas con riesgo de manifestaciones severas).

\section{Enfermedad crítica}

Enfermedad de rápida progresión hacia el deterioro acompañada de:

- Fallo respiratorio y necesidad de ventilación mecánica debido a hipoxia permanente que no mejora con cánula nasal o mascarilla con oxígeno o síndrome agudo respiratorio severo.

- Encefalopatía, shock séptico, daño miocárdico, fallo cardíaco, encefalopatía.

- $\quad$ Enfermedad de Kawasaki, síndrome inflamatorio multisistémico.

- Fallo multiorgánico.

- Hallazgos de laboratorio similares a los de las manifestaciones severas + elevación de enzimas hepáticas.

Nota. Referencias (de Souza et al., 2020; Gil et al., 2020; Lot et al., 2020; Qiu et al., 2020). 
Tabla 2

Tratamiento para niños en casos leves y moderados de COVID-19

\section{Casos leves}

Pacientes sin dificultad respiratoria, con saturación de oxígeno $>92 \%$ :

- Ingreso a área de aislamiento hospitalaria si existe disponibilidad o aislamiento en el hogar, con seguimiento por telemedicina.

- Uso de antibióticos si hay síntomas de neumonía leve cubriendo gérmenes comunitarios (amoxicilina clavulanato: $50 \mathrm{mg} / \mathrm{kg}$ ).

Tratamiento de sostén:

- Antipirético y analgésico paracetamol (10-15 mg/kg/ dosis).

- Hidratación, educación a padres sobre datos de alerta y uso apropiado de equipo de protección personal (EPP).

\section{Casos moderados}

Medidas generales y monitoreo de signos vitales.

Mantener aporte de oxígeno para alcanzar saturación $>92 \%$, uso secuencial de cánula binasal a cánula de alto flujo. Manejo de líquidos a 70-80\% de requerimientos si no hay datos de hipovolemia.

Antipirético y analgésico paracetamol (10-15 mg/kg/dosis).

Toma de hemocultivo.

Inicio de antibiótico (ceftriaxona $100 \mathrm{mg} / \mathrm{kg} /$ día) si hay datos de sepsis o choque séptico.

Fármacos inhalados (esteroides, beta agonistas de acción corta) si hay datos de obstrucción bronquial, con uso de MDI para minimizar aerosoles.

Nota. Referencias: (Calvo et al., 2020; Sankar et al., 2020).

Se espera que se diseñen estudios específicos que consideren los beneficios directos y los riesgos aceptables, para iniciar las aplicaciones de las nuevas vacunas en población pediátrica, evaluando eficacia y seguridad y considerando la diversidad de las manifestaciones clínicas (U.S. Department of Health and Human Services et al., 2020).

\section{Prevención}

Para que los niños no enfermen, es necesario que los padres estén informados de la forma de transmisión de la enfermedad, que den el ejemplo cumpliendo las acciones preventivas como el lavado de manos y el uso correcto de la mascarilla y que les expliquen de forma sencilla y de acuerdo con su edad, qué es la enfermedad, por qué no pueden salir a jugar con otros niños y por qué deben evitar abrazar y acercarse a familiares, estén o no estén enfermos.
Para prevenir la dispersión de la enfermedad hay que evitar que el niño tenga contacto con otras personas, especialmente si son adultos mayores o con enfermedades crónicas y procurar que salga de su domicilio lo menos posible. A partir de los 2 años, se les puede explicar el uso correcto de la mascarilla y colocarles una bajo supervisión de un adulto responsable, evitando el uso de mascarilla en los menores de 2 años. La limpieza y desinfección de la casa, las superficies y los juguetes de los niños es de particular importancia para evitar el contagio (CDC, 2020b; Mayo Clinic, 2020).

Con los adolescentes es importante mantener una comunicación adecuada, darles el ejemplo con las medidas de prevención y el cumplimiento de las normas que dicte el gobierno local para contener la pandemia, así como proporcionar información veraz y adecuada acerca del virus y las recomendaciones concretas acerca de cómo prevenir su propagación. Se recomienda 
Tabla 3

Esquema de seguimiento en casos graves de COVID-19

Ventilación mecánica: vigilar frecuencia y esfuerzo respiratorio.

- Ventilación no invasiva (Prevención de contagio por generación de aerosoles).

- Ventilación invasiva si el paciente no tolera ventilación no invasiva, hay inestabilidad hemodinámica, hipoxemia o incremento de trabajo respiratorio.

- Usar ventilación de protección pulmonar (volúmenes tidal bajos (4-8 ml/kg), PEEP óptima, presión meseta $\leq 28-32 \mathrm{cmH}_{2} \mathrm{O}$, driving pressure $<15 \mathrm{cmH}_{2} \mathrm{O}$, hipercapnia permisiva, etc.).

- Usar tempranamente ventilación prono.

- Considerar ECMO (extracorporeal membrane oxigenation).

Hemodinamia: monitoreo de signos vitales y datos de perfusión distal:

- Reconocimiento temprano de choque.

- $\quad$ Soporte hídrico y vasopresor según la campaña de sobrevivencia a la sepsis.

Antimicrobianos: no existe agente especifico aprobado/ no abusar de la prescripción

Lopinavir/itonavir.

Remdesivir.

- Es un profármaco antiviral análogo de nucleótido. Las dosis propuestas para la población pediátrica son:

- $\quad \geq 40 \mathrm{~kg}$ de peso: el primer día de $200 \mathrm{mg} / \mathrm{iv}$ seguido de una dosis de mantenimiento de $100 \mathrm{mg} / \mathrm{iv}$ al día desde el día 2 al día 10.

- $\quad<40 \mathrm{Kg}$ de peso: dosis primer día de $5 \mathrm{mg} / \mathrm{kg}$ iv seguido de una dosis de mantenimiento de $2.5 \mathrm{mg} / \mathrm{kg}$ iv al día desde el día 2 al día 9.

Antibióticos según patología bacteriana o viral confirmada.

Reanimación: Los reanimadores requieren uso estricto de Equipo de Protección Personal (EPP).

- Minimizar la cantidad de personal expuesto.

- Colocar filtro en la bolsa válvula mascarilla (ambu®).

\section{Manejo inmunológico}

Interferón $\alpha$ (200,000-400,000 IU/kg o 2-4 $\mu \mathrm{g} / \mathrm{kg}$ en $2 \mathrm{~mL}$ de agua estéril cada 12 por 5-7 días).

Esteroides: metilprednisolona 1-2 mg/kg/día (en casos de síndrome de dificultad respiratoria aguda SDRA o miocardio depresión).

Inmunoglobulina: (miocardio depresión, encefalitis, síndrome hemo fagocítico, trastorno inflamatorio multisistémico).

Antagonistas de IL-6: Tocilizumab (400 mg diluido en solución salina para completar $100 \mathrm{ml}$ ), durante $1 \mathrm{~h}$ (máximo dos dosis).

\section{Medidas generales}

Mantener ingesta calórica.

Mantener equilibrio ácido-base y electrolitos.

\section{Laboratorios}

Hemograma, química sanguínea, tiempos de coagulación, dímero D, proteína C reactiva, IL-6, pruebas de función renal y hepática, panel cardiaco (troponinas, BNP), gasometría arterial y venosa, cultivos.

Nota. Referencias: (Antonucci, et al., 2014; Calvo, et al., 2020; Dixon, et al., 2020; Echeverri, et al., 2017; Hunter \& Doddi, 2010; Jouvet et al., 2015; Kache, et al., 2020; Liguoro, et al., 2020; Lotfi, et al., 2020; Matthay, et al., 2018; Price et al., 2020; Rothan \& Byrareddy, 2020; Sankar, et al., 2020; Sanna, et al., 2020; Shen, et al., 2020; Szabari, et al., 2019; World Health Organization, 2020b; Zimmermann \& Curtis, 2020). 
Tabla 4

Propuestas de tratamiento de trastorno inflamatorio multisistémico

- OMS/1 $\lambda$ : activación de células epiteliales, reducción de reclutamiento de neutrófilos, no hay datos sobre reducción significativa de la mortalidad.

- Cloroquina: inhibidor de producción y liberación de TNF e IL-6, no útil por el incremento de mortalidad y efectos adversos cardiovasculares.

- Ulinastatina: producto endógeno que reduce los niveles de factores proinflamatorios TNF- $\alpha$, IL-6 e IFN- $\gamma$ e induce el incremento de IL-10.

- $\quad$ Procesos de captación de citoquinas: Citosorb $®$, aun no utilizado en niños.

- $\quad$ Antagonista de IL-1: Anakinra, no existe experiencia en el tratamiento de COVID-19, al momento de la preparación de esta revisión.

Nota. Referencia: (Lega et al., 2020).

utilizar plataformas digitales de sitios con información oficial y científicamente validada (Fondo de Población de las Naciones Unidas, 2020).

En relación con la contaminación durante el periodo perinatal, Zullo y colaboradores (2020) han propuesto un algoritmo para realizar tamizaje con prueba rápida de anticuerpos $\operatorname{IgM}-\operatorname{IgG}$ y si una o ambas salen positivas, aislar a la paciente hasta confirmar con PCR-RT. Esto permitiría detectar los casos positivos y manejarlos adecuadamente, disminuyendo el contagio a los neonatos y al personal de salud, en pacientes que ingresan por emergencia obstétrica. Otros autores han propuesto que es mejor considerar como positivas a todas las pacientes obstétricas, pues los falsos negativos para PCR-RT en muestras nasofaríngeas oscilan entre 17 y $63 \%$ (Kelly et al., 2020).

\section{Consideraciones y cuidados especiales en recién nacidos asintomáticos y con infección leve}

En cuanto a los efectos perinatales del COVID-19, una revisión sistemática y un metaanálisis concluyeron que la mayoría de recién nacidos no presenta síntomas, o son leves. Las complicaciones perinatales detectadas en recién nacidos con prueba positiva fueron síndrome de distrés respiratorio, neumonía con fiebre, asfixia perinatal, estrés fetal intrauterino, nacimiento pretérmino, restricción del crecimiento intrauterino y líquido amniótico con meconio. No se reportaron casos de asfixia severa ni muerte (Di Mascio et al., 2020; Duran et al., 2020).
Según los resultados de la revisión sistemática de Durán y colaboradores (2020), el corte retardado del cordón, el contacto piel con piel y la lactancia materna pueden continuar si la madre con diagnóstico o sospecha de COVID-19 está en condiciones de cuidar al neonato. Se recomienda el uso de una mascarilla quirúrgica siempre que la madre esté con su hijo, que realice una adecuada higiene de manos y de mamas antes de alimentarlo y use una bata de algodón cada vez que amamante. Si no está en condiciones de cuidarlo, se recomienda que se extraiga la leche, conservando las normas de higiene, para evitar contaminación y alimentar al niño usando cuchara o paladai. Si la madre ha salido de cuidados intensivos, es necesario revisar los medicamentos que ha consumido antes de aconsejar la extracción de leche. La WHO (2020d) realizó una revisión sistemática y concluyó que los beneficios de la lactancia materna y la interacción nutritiva madre-hijo para prevenir las infecciones y promover la salud y el desarrollo del niño, deben ser preservados. El aislamiento del neonato al momento de nacer, solo se aconseja si la madre está críticamente enferma (Boelig et al., 2020; CDC, 2020a; Centro Nacional de Equidad de Género y Salud Reproductiva [CNEGSR], 2020; Ministerio de Salud de Costa Rica, 2020; Naranje et al., 2020).

Tanto la madre como su hijo (sea o no positivo), requieren monitorización inmediata al nacimiento y vigilancia continua con seguimiento domiciliar. Si la madre está en condiciones de cuidar a su hijo, deben permanecer en un cuarto aislado de otras personas con adecuada ventilación y se debe colocar al recién 
nacido en cuna, a $1.8 \mathrm{~m}$ de distancia de la cama de la madre. Los pañales deben ser cambiados con guantes y descartados como material bioinfeccioso, pues se sospecha que los niños pueden excretar virus por las heces, aunque sus hisopados nasofaríngeos sean negativos (CDC, 2020b; Ministerio de Salud de Costa Rica, 2020; Naranje et al., 2020; Zhang et al., 2020).

\section{Conclusiones}

La infección por SARS-CoV-2 se ha convertido en la pandemia de mayor impacto para la salud pública de todo el mundo desde 1918. Han pasado cerca de 8 meses desde el reconocimiento del primer caso de COVID-19, los datos aún son dispersos y no estandarizados, lo que no permite contar con referentes sólidos sobre el comportamiento de la enfermedad en niños. Las manifestaciones en menores de 19 años han sido reconocidas como más leves que en los adultos, sin embargo, hay registros de niños hospitalizados en cuidados intensivos y fallecidos a causa de esta enfermedad.

Hace falta investigación para comprender qué tan importante es la participación de los niños en la transmisión del SARS-CoV-2, así como datos de seroprevalencia específicos por edad para contar con información de la dinámica de transmisión y de los niveles de inmunidad de la población para diseñar programas de vigilancia epidemiológica y revisión de las políticas de salud.

No existe ningún medicamento específico científicamente validado para el tratamiento de COVID-19. Actualmente en niños, se toman decisiones terapéuticas basadas en experiencias del tratamiento en adultos, particularmente en los casos severos que se han descrito como un síndrome inflamatorio multisistémico asociado temporalmente a SARS-CoV-2, para lo que se recomienda el abordaje a través de un equipo médico multidisciplinario integrado por pediatras especialistas en cuidado crítico, infectólogos, reumatólogos y cardiólogos entre otros.

\section{Referencias}

Antonucci, E., Fiaccadori, E., Donadello, K., Taccone, F. S., Franchi, F., \& Scolletta, S. (2014). Myocardial depression in sepsis: From pathogenesis to clinical manifestations and treatment. Journal of Critical Care, 29(4), 500511. https://doi.org/10.1016/j.jcrc.2014.03.028
Balasubramanian, S., Rao, N. M., Goenka, A., Roderick, M., \& Ramanan, A. V. (2020). Coronavirus disease 2019 (COVID-19) in children - What we know so far and what we do ot. Indian Pediatrics, 57(5), 435-442. https://doi. org/10.1007/s13312-020-1819-5

Boelig, R. C., Manuck, T., Oliver, E. A., Di Mascio, D., Saccone, G., Bellussi, F., \& Berghella, V. (2020). Labor and delivery guidance for COVID-19. American Journal of Obstetrics \& Gynecology MFM, 2(2), Article e100110. https:// doi.org/10.1016/j.ajogmf.2020.100110

Calvo, C., Garcia López-Hortelano, M., Vicente, J. C., \& Martínez Vázquez, J. L. (2020). Recomendaciones sobre el manejo clínico de la infección por el «nuevo coronavirus» SARS-CoV2. Grupo de trabajo de la Asociación Española de Pediatría (AEP). Anales de Pediatria, 92(4), 241.el-241e11. https://doi.org/10.1016/j.anpedi.2020.02.001

Castagnoli, R., Votto, M., Licari, A., Brambilla, I., Bruno, R., Perlini, S., ... Marseglia, G. L. (2020). Severe acute respiratory syndrome coronavirus 2 (SARS-CoV-2) infection in children and adolescents: A systematic review. JAMA Pediatrics, 174(9), 882-889. https://doi. org/10.1001/jamapediatrics.2020.1467

Centers for Disease Control and Prevention. (2020a). Coronavirus Disease 2019 (COVID-19): Evaluation and management considerations for neonates at risk for COVID-19. Recuperado el 22 de julio de 2020 de https://www.cdc.gov/coronavirus/2019ncov/hcp/caring-for-newborns.html

Centers for Disease Control and Prevention. (2020b). Mantenga sanos a los niños durante el brote de COVID-19. Recuperado el 22 de julio de 2020 de https://espanol.cdc.gov/coronavirus/2019-ncov/ daily-life-coping/children.html

Centro Nacional de Equidad de Género y Salud Reproductiva. (2020). Lineamiento para la prevención y mitigación de COVID-19 en la atención del embarazo, parto, puerperio y de la persona recién nacida. Secretaría de Salud. Recuperado de https://www.gob.mx/salud/ cnegsr/prensa/lineamiento-para-la-prevenciony-mitigacion-de-covid-19-en-la-atencion-delembarazo-parto-puerperio-y-de-la-personarecien-nacida?idiom $=\mathrm{es}$ 
Cruz, A. T., \& Zeichner, S. L. (2020). COVID-19 in Children: Initial characterization of the pediatric disease Andrea. Pediatrics, 145(6), 6-10. https:// doi.org/https://doi.org/10.1542/peds.2020-0834

de Souza, T. H., Nadal, J. A., Nogueira, R. J. N., Pereira, R. M., \& Brandão, M. B. (2020). Clinical manifestations of children with COVID-19: A systematic review. Pediatric Pulmonology, 55, 1892-1899. https://doi.org/10.1002/ppul.24885

Dhochak, N., Singhal, T., Kabra, S. K., \& Lodha, R. (2020). Pathophysiology of COVID-19: Why children fare better than adults? Indian Journal of Pediatrics, 87(7), 537-546. https://doi. org/10.1007/s12098-020-03322-y

Di Mascio, D., Khalil, A., Saccone, G., Rizzo, G., Buca, D., Liberati, M., ... D’Antonio, F. (2020). Outcome of coronavirus spectrum infections (SARS, MERS, COVID-19) during pregnancy: A systematic review and meta-analysis. American Journal of Obstetrics \& Gynecology MFM, 2(2), Article e100107. https://doi.org/10.1016/j. ajogmf.2020.100107

Dixon, D. L., Van Tassell, B. W., Vecchié, A., Bonaventura, A., Talasaz, A., Kakavand, H., ... Abbate, A. (2020). Cardiovascular considerations in treating patients with coronavirus (COVID-19). Journal of Cardiovascular Pharmacology, 75(5), 359-367. https://doi.org/10.1097/ FJC.0000000000000836

Duran, P., Berman, S., Niermeyer, S., Jaenisch, T., Forster, T., Gomez, R., ... Serruya, S. (2020). COVID-19 and newborn health: systematic review. Revista Panamericana de Salud Pública, 44, 1. https://doi.org/10.26633/rpsp.2020.54

Echeverri, D., Fontanilla, M., Buitrago, L., Bruchfeld, A., Carrero, J. J., Qureshi, A. R., ... Xia, X. Z. (2017). The surviving sepsis campaign. Molecular Immunology, 44(2), 266-277. https:// doi.org/10.1097/01.shk.0000246898.65692.34

European Centre for Disease Prevention and Control. (2020a). Epidemiology of COVID-19, Demographic profiles Children. Recuperado de https://www.ecdc.europa.eu/en/covid-19/latestevidence/epidemiology

European Centre for Disease Prevention and Control. (2020b). Epidemiology of COVID-19. Epidemiology of COVID-19 1. Recuperado de https://www.ecdc.europa.eu/en/covid-19/latestevidence/epidemiology.

Felsenstein, S., Herbert, J. A., Mcnamara, P. S., \& Hedrich, C. M. (2020). COVID-19: Immunology and treatment options. Clinical Immunology, 215, 108448. https://doi.org/10.1016/j. clim.2020.108448

Fondo de Población de las Naciones Unidas. (24 de marzo de 2020). Los adolescentes y los jóvenes y la enfermedad del coronavirus (COVID-19). Recuperado de https://www.unfpa.org/sites/ default/files/resource-pdf/COVID19-TechBriefYouth-24Mar20.pdf

Gasparyan, A. Y., Misra, D. P., Yessirkepov, M., \& Zimba, O. (2020). Perspectives of immune therapy in coronavirus disease 2019. Journal of Korean Medical Science, 35(18), Article e176. https://doi.org/10.3346/JKMS.2020.35.E176

Gil, R., Jacinto, L., Lemus, P., Barillas, G., Velásquez, S., \& Recinos, F. (2020). Guía de diagnóstico clínico y manejo de pacientes pediátricos sospechosos y confirmados de COVID-19. Instituto Guatemalteco de Seguridad Social. Recuperado el 22 de julio de 2020 en https://www. igssgt.org/wp-content/uploads/2020/05/Guia-deDiagnostico-y-Manejo-de-Pacientes-PediatricosSospechosos-y-confirmados-COVID-19.pdf

Gottlieb, M., \& Long, B. (2020). Dermatologic manifestations and complications of COVID-19. American Journal of Emergency Medicine, 38(9), 1715-1721. https://doi.org/10.1016/j. ajem.2020.06.011

Götzinger, F., Santiago-García, B., Noguera-Julián, A., Lanaspa, M., Lancella, L., Calò Carducci, F. I., ... Riordan, A. (2020). COVID-19 in children and adolescents in Europe: a multinational, multicentre cohort study. The Lancet Child \& Adolescent Health, 4642(20), 1-9. https://doi. org/10.1016/s2352-4642(20)30177-2

He, Y., Wang, J., Li, F., \& Shi, Y. (2020). Main clinical features of COVID-19 and potential prognostic and therapeutic value of the microbiota in SARSCoV-2 infections. Frontiers in Microbiology, 11(1302), 1-7. https://doi.org/10.3389/ fmicb.2020.01302

Henry, B. M., De Oliveira, M. H. S., Benoit, S., Plebani, M., \& Lippi, G. (2020). Hematologic, biochemical 
and immune biomarker abnormalities associated with severe illness and mortality in coronavirus disease 2019 (COVID-19): A meta-analysis. Clinical Chemistry and Laboratory Medicine, 58(7), 1021-1028. https://doi.org/10.1515/cclm2020-0369

Hong, H., Wang, Y., Chung, H. T., \& Chen, C. J. (2020). Clinical characteristics of novel coronavirus disease 2019 (COVID-19) in newborns, infants and children. Pediatrics and Neonatology, 61(2), 131-132. https://doi.org/10.1016/j. pedneo.2020.03.001

Hunter, J. D., \& Doddi, M. (2010). Sepsis and the heart. British Journal of Anaesthesia, 104(1), 3-11. https://doi.org/10.1093/bja/aep339

Hwang, T. J., Randolph, A.G., \& Bourgeois, F. T. (2020). Inclusion of children in clinical trials of treatments for Coronavirus disease 2019 (COVID-19). JAMA Pediatrics, 174(9), 825-826. https://doi.org/10.1001/jamapediatrics.2020. 1888

Jin, Y., Yang, H., Ji, W., Wu, W., Chen, S., Zhang, W., \& Duan, G. (2020). Virology, epidemiology, pathogenesis, and control of covid-19. Viruses, 12(4), 1-17. https://doi.org/10.3390/v12040372

Jouvet, P., Thomas, N. J., Willson, D. F., Erickson, S., Khemani, R., Smith, L., ... Bembea, M. (2015). Pediatric acute respiratory distress syndrome: Consensus recommendations from the pediatric acute lung injury consensus conference. Pediatric Critical Care Medicine, 16(5), 428-439. https:// doi.org/10.1097/PCC.0000000000000350

Kache, S., Chisti, M. J., Gumbo, F., Mupere, E., Zhi, X., Nallasamy, K., ... Carcillo, J. (2020). COVID-19 PICU guidelines: For high- and limited-resource settings. Pediatric Research. https://doi.org/10.1038/s41390-020-1053-9

Kelly, J. C., Dombrowksi, M., O’Neil-Callahan, M., Kernberg, A. S., Frolova, A. I., \& Stout, M. J. (2020). False-negative testing for severe acute respiratory syndrome coronavirus 2: Consideration in obstetrical care. American Journal of Obstetrics \& Gynecology MFM, Article e100130. https://doi.org/10.1016/j. ajogmf.2020.100130

Lega, S., Naviglio, S., Volpi, S., \& Tommasini, A. (2020). Recent insight into SARS-COV2 immunopathology and rationale for potential treatment and preventive strategies in COVID-19. Vaccines, 8(2), 1-30. https://doi.org/10.3390/ vaccines 8020224

Li, H., Liu, L., Zhang, D., Xu, J., Dai, H., Tang, N., ... Cao, B. (2020). SARS-CoV-2 and viral sepsis: observations and hypotheses. Lancet, 2019(20), 8-11. https://doi.org/10.1016/S01406736(20)30920-X

Liguoro, I., Pilotto, C., Bonanni, M., Ferrari, M. E., Pusiol, A., Nocerino, A., ... Cogo, P. (2020). SARS-COV-2 infection in children and newborns: A systematic review. European Journal of Pediatrics, 179(7), 1029-1046. https:// doi.org/10.1007/s00431-020-03684-7

Lin, L., Lu, L., Cao, W., \& Li, T. (2020). Hypothesis for potential pathogenesis of SARS-CoV-2 infection-a review of immune changes in patients with viral pneumonia. Emerging Microbes and Infections, 9(1), 727-732. https://doi.org/10.1080/ 22221751.2020.1746199

Lotfi, M., Hamblin, M. R., \& Rezaei, N. (2020). COVID-19: Transmission, prevention, and potential therapeutic opportunities. Clinica Chimica Acta, 508, 254-266. https://doi. org/10.1016/j.cca.2020.05.044

Ludvigsson, J. F. (2020). Systematic review of COVID-19 in children shows milder cases and a better prognosis than adults. Acta Paediatrica, International Journal of Paediatrics, 109(6), 1088-1095. https://doi.org/10.1111/apa.15270

Maggi, E., Canonica, W., \& Moretta, L. (2020). COVID-19: Unanswered questions on immune response and pathogenesis. Journal of Allergy Clinical Immunology, 146(1), 18-22. https://doi. org/10.1016/j.jaci.2020.05.001

Mantovani, A., Rinaldi, E., Zusi, C., Beatrice, G., Saccomani, M. D., \& Dalbeni, A. (2020). Coronavirus disease 2019 (COVID-19) in children and/or adolescents: A meta-analysis. Pediatric Research. https://doi.org/10.1038/ s41390-020-1015-2

Matthay, M. A., Zemans, R. L., Zimmerman, G. A., Arabi, Y. M., Beitler, J. R., Mercat, A., ... Calfee, C. S. (2018). Acute respiratory distress syndrome. Nature Reviews Disease Primers, 5(1). https://doi. org/10.1038/s41572-019-0069-0 
Mayo Clinic. (2020). COVID-19 (coronavirus) en bebés y niños. Recuperado de https://www.mayoclinic.or g/es-es/diseases-conditions/coronavirus/in-depth/ coronavirus-in-babies-and-children/art-20484405

Ministerio de Salud de Costa Rica. (2020). Lineamientos generales sobre lactancia materna y COVID-19. Recuperado de https://www.ministeriodesalud. go.cr/sobre_ministerio/ prensa/docs/lactancia_ materna_v1_19032020.pdf

Ministerio de Salud Pública y Asistencia Social. (2020). Situación de COVID-19 en Guatemala. Recuperado el 22 julio 2020 de https:// tablerocovid.mspas.gob.gt/

Moore, J. B., \& June, C. H. (2020). Cytokine release syndrome in severe COVID-19. Science, 368(6490), 473-474. https://doi.org/10.1126/ science.abb8925

Naranje, K. M., Gupta, G., Singh, A., Bajpai, S., Verma, A., Jaiswal, R., ... Birthare, A. (2020). Neonatal COVID-19 infection management. Journal of Neonatology, 32(1-2), 88-98. https:// doi.org/10.1177/0973217920928638

Nguyen, A. A., Habiballah, S. B., Platt, C. D., Geha, R. S., Chou, J. S., \& Mcdonald, D. R. (2020). Immunoglobulins in the treatment of COVID-19 infection: Proceed with caution! Clinical Immunology, 216, Article e108459. https://doi. org/10.1016/j.clim.2020.108459.

Patel, N. A. (2020). Pediatric COVID-19: Systematic review of the literature. American Journal of Otolaryngology, 41(5), Article e102573. https:// doi.org/10.1016/j.amjoto.2020.102573

Price, S., Singh, S., Ledot, S., Bianchi, P., Hind, M., Tavazzi, G., \& Vranckx, P. (2020). Respiratory management in severe acute respiratory syndrome coronavirus 2 infection. European Heart Journal. Acute Cardiovascular Care, 9(3) 229-238. https:// doi.org/10.1177/2048872620924613

Qiu, H., Wu, J., Hong, L., Luo, Y., Song, Q., \& Chen, D. (2020). Clinical and epidemiological features of 36 children with coronavirus disease 2019 (COVID-19) in Zhejiang, China: An observational cohort study. The Lancet Infectious Diseases, 20(6), 689-696. https://doi.org/10.1016/ S1473-3099(20)30198-5

Rasmussen, S. A., Smulian, J. C., Lednicky, J. A., Wen, T. S., \& Jamieson, D. J. (2020). Coronavirus
Disease 2019 (COVID-19) and pregnancy: What obstetricians need to know. American Journal of Obstetrics and Gynecology, 222(5), 415-426. https://doi.org/10.1016/j.ajog.2020.02.017

Rehman, S., Majeed, T., Azam, M., Ali, U., \& Sabit, H. (2020). Current scenario of COVID-19 in pediatric age group and physiology of immune and thymus response. Saudi Journal of Biological Sciences, 27(10), 2567-2573. https:// doi.org/10.1016/j.sjbs.2020.05.024

Riggioni, C., Giovannini, M., Agache, Ioana., Akdis, M,. Alves-Correia, M., ... Akdis, C. A. (2020). A compendium answering 150 questions on COVID-19 and SARS-CoV-2. Allergy. 1-39. https://doi.org/10.1111/all.14449

Rothan, H. A., \& Byrareddy, S. N. (2020). The epidemiology and pathogenesis of coronavirus disease (COVID-19) outbreak. Journal of Autoimmunity, 109, Article e102433. https://doi. org/10.1016/j.jaut.2020.102433

Safadi-Palazzi, M. A. (2020). The intriguing features of COVID-19 in children and its impact on the pandemic. Jornal de Pediatria, 96(3), 265-268. https://doi.org/10.1016/j.jped.2020.04.001

Sankar, J., Dhochak, N., Kabra, S. K., \& Lodha, R. (2020). COVID-19 in children: Clinical approach and management. Indian Journal of Pediatrics, 87(6), 433-442. https://doi.org/10.1007/s12098020-03292-1

Sanna, G., Serra, G, Bassareo, P. P., Neroni, P., Fanos, V., \& Marcialis, M. A. (2020). Children's heart and COVID-19: Up-to-date evidence in the form of a systematic review. European Journal of Pediatrics, 179, 1079-1087. https://doi. org/10.1007/s00431-020-03699-0

She, J., Liu, L., \& Liu, W. (2020). COVID-19 epidemic: Disease characteristics in children. Journal of Medical Virology, 92(7), 747-754. https://doi. org/10.1002/jmv.25807.

Shen, K.-L., Yang, Y.-H., Jiang, R-M., Wang, T.Y., Zhao, D.-C., Jiang, Y., ... Global Pediatric Pulmonology Alliance (2020). Updated diagnosis, treatment and prevention of COVID-19 in children: Experts' consensus statement (condensed version of the second edition). World Journal of Pediatrics, 16(3), 232-239. https://doi. org/10.1007/s12519-020-00362-4 
Szabari, M. V., Takahashi, K., Feng, Y., Locascio, J. J., Chao, W., Carter, E. A., ... Musch, G. (2019). Relation between respiratory mechanics, inflammation, and survival in experimental mechanical ventilation. American Journal of Respiratory Cell and Molecular Biology, 60(2), 179-188. https://doi.org/10.1165/rcmb.2018$01000 \mathrm{C}$

Tian, D., \& Ye, Q. (2020). Hepatic complications of COVID-19 and its treatment. Journal of Medical Virology, 92(10), 1818-1824. https://doi. org/10.1002/jmv.26036

Uddin, M., Mustafa, F., Rizvi, T. A., Loney, T., Al Suwaidi, H.., Al-Marzouqi, H., ... Senok, A. C. (2020). SARS-CoV-2/COVID-19: Viral genomics, epidemiology, vaccines, and therapeutic interventions. Viruses, 10, 526. https://doi.org/10.3390/v12050526

U.S. Department of Health and Human Services, Food and Drug Administration, \& Center for Biologics Evaluation and Research. (2020). Development and Licensure of Vaccines to Prevent COVID-19. Guidance for Industry. (FDA-2020-D-1137). Recuperado de https:// www.fda.gov/media/139638/download

Velásquez-Gómez, L., Pinillos-Montenegro, N., Calderón-Rivera, V., Álvarez-Castañeda, A., \& Gómez-Hernández, V. (2020). Guía de consenso para el manejo de COVID-19 en neonatos (V.0215/06/20). Instituto Guatemalteco de Seguridad Social. Recuperado de https://www.igssgt.org/wpcontent/uploads/2020/05/Guia-de-consenso-parael-manejo-de-COVID-19-en-neonatos.pdf?=137

Wang, H., \& Ma, S. (2008). The cytokine storm and factors determining the sequence and severity of organ dysfunction in multiple organ dysfunction syndrome. American Journal of Emergency Medicine, 26(6), 711-715. https://doi.org/10.1016/j. ajem.2007.10.031

Wollina, U., Karadağ, A. S., Rowland-Payne, C., Chiriac, A., \& Lotti, T. (2020). Cutaneous signs in COVID-19 patients: A review. Dermatologic Therapy, 2020, Article e13549, 1-6. https://doi. org/10.1111/dth.13549

World Health Organization (2020a) Management of Covid-19, Interim Guidance, 27 May 2020. World Health Organization. https://apps.who.int/iris/ handle/10665/332638. License: CC BY-NC-SA $3.0 \mathrm{IGO}$

World Health Organization. (15 May 2020b). Multisystem inflammatory syndrome in children and adolescents with COVID-19. Scientific brief. https:/www.who.int/news-room/commentaries/ detail/multisystem-inflammatory-syndrome-inchildren-and-adolescents-with-covid-19

World Health Organization. (2020c). Draft landscape of COVID-19 candidate vaccines. Recuperado el 15 May 2020, de https://www.who.int/ publications/m/item/draft-landscape-of-covid19-candidate-vaccines

World Health Organization. (2020d). Breastfeeding and COVID-19. (WHO/2019-nCoV/SciBrief/ Breastfeeding/ 2020). Recuperado de: https:// www.who.int/ publications/i/item/WHO-2019nCoV-Sci_Brief-Breastfeeding-2020.1

Yong, S. E. F., Anderson D. A. Wei, W. E., Pang, J., Chia, W. N., Tang, C. H., ...Lee, V. J. M. (2020). Connecting clusters of COVID-19: An epidemiological and serological investigation. The Lancet Infectious Diseases, 20(7), 809-815. https://doi.org/10.1016/S1473-3099(20)30273-5

Yuki, K., Fujiogi, M., \& Koutsogiannaki, S. (2020). COVID-19 pathophysiology: A review. Clinical Immunology, 215. https://doi.org/10.1016/j. clim.2020.108427

Zhang, B., Liu, S., Dong, Y., Zhang, L., Zhong, Q., Zou, Y., \& Zhang, S. (2020). Positive rectal swabs in young patients recovered from coronavirus disease 2019 (COVID-19). Journal of Infection, 81(2), 13-37. https://doi.org/10.1016/j. jinf.2020.04.023

Zimmermann, P., \& Curtis, N. (2020). Coronavirus infections in children including COVID-19: An overview of the epidemiology, clinical features, diagnosis, treatment and prevention options in children. Pediatric Infectious Disease Journal, 39(5), 355-368. https://doi.org/10.1097/ INF.0000000000002660

Zullo, F., Di Mascio, D., \& Saccone, G. (2020). COVID-19 antibody testing in pregnancy. American Journal of Obstetrics \& Gynecology MFM, 2(3), 100142. https://doi.org/10.1016/j. ajogmf.2020.100142 